\title{
Hepatoblastoma and Wilms' tumour in an infant with Beckwith-Wiedemann syndrome and diazoxide resistant congenital hyperinsulinism
}

\author{
Saurabh Uppal1,*, James Blackburn'1,*, Mohammed Didi1, Rajeev Shukla², \\ James Hayden ${ }^{3}$ and Senthil Senniappan ${ }^{1,4}$ \\ Departments of ${ }^{1}$ Paediatric Endocrinology, 2 Pathology, ${ }^{3}$ Oncology, Alder Hey Children's NHS Foundation Trust, \\ Liverpool, UK, and 4Institute of Child Health, University of Liverpool, Liverpool, UK \\ *(S Uppal and J Blackburn contributed equally to this work)
}

Correspondence should be addressed to S Senniappan Email

senthilkss@yahoo.co.uk

\section{Summary}

Beckwith-Wiedemann syndrome (BWS) can be associated with embryonal tumours and congenital hyperinsulinism (CHI). We present an infant with BWS who developed congenital hepatoblastoma and Wilms' tumour during infancy. The infant presented with recurrent hypoglycaemia requiring high intravenous glucose infusion and was biochemically confirmed to have $\mathrm{CHI}$. He was resistant to diazoxide but responded well to octreotide and was switched to Lanreotide at 1 year of age. Genetic analysis for mutations of $A B C C 8$ and $K C N J 11$ were negative. He had clinical features suggestive of BWS. Methylation-sensitive multiplex ligation-dependent probe amplification revealed hypomethylation at KCNQ1OT1:TSS-DMR and hypermethylation at H19/IGF2:IG-DMR consistent with mosaic UPD(11p15). Hepatoblastoma was detected on day 4 of life, which was resistant to chemotherapy, requiring surgical resection. He developed Wilms' tumour at 3 months of age, which also showed poor response to induction chemotherapy with vincristine and actinomycin D. Surgical resection of Wilms' tumour was followed by post-operative chemotherapy intensified with cycles containing cyclophosphamide, doxorubicin, carboplatin and etoposide, in addition to receiving flank radiotherapy. We report, for the first time, an uncommon association of hepatoblastoma and Wilms' tumour in BWS in early infancy. Early onset tumours may show resistance to chemotherapy. UPD(11p15) is likely associated with persistent $\mathrm{CHI}$ in BWS.

\section{Learning points:}

- Long-acting somatostatin analogues are effective in managing persistent CHI in BWS.

- UPD(11)pat genotype may be a pointer to persistent and severe $\mathrm{CHI}$.

- Hepatoblastoma and Wilms' tumour may have an onset within early infancy and early tumour surveillance is essential.

- Tumours associated with earlier onset may be resistant to recognised first-line chemotherapy.

\section{Background}

Beckwith-Wiedemann syndrome (BWS) is a human genomic imprinting disorder characterised by overgrowth, tumour predisposition and congenital malformations. Typical clinical features include macroglossia, abdominal wall defects, hemihyperplasia (lateralized overgrowth), enlarged abdominal organs 
and an increased risk of embryonal tumours during early childhood.

The molecular aetiology of BWS involves genetic and epigenetic changes at chromosome 11p15.5. The involvement of genes regulating foetal growth, such as CDKN1C and IGF2, which are present at this locus, explains several aspects of the phenotypic expression. About $50 \%$ of patients with BWS present with hypoglycaemia secondary to hyperinsulinism of varying severity. Prolonged and severe hyperinsulinism is seen in up to $20 \%$ of cases (1).

Embryonal tumours occur in $7.5-10 \%$ of children with BWS (2). Alterations in tumour suppressor genes and growth factor-related genes on the chromosome locus 11p15.5 might account for a significant proportion of these neoplasms. This case uniquely describes the occurrence of very early onset hepatoblastoma and Wilms' tumour. The case highlights the need for early screening and identification of tumours in patients with BWS. The treatment resistance of both hepatoblastoma and Wilms' tumour suggest that embryonal tumours, which present early, may be resistant to standard treatment regimens.

We report a case of BWS secondary to paternal uniparental disomy of chromosome 11 (UPD(11)pat) with diazoxide-resistant congenital hyperinsulinism (CHI) and early onset hepatoblastoma and Wilms' tumour.

\section{Case presentation}

The patient was born to non-consanguineous white Caucasian parents at 36 weeks gestation via elective lower segment caesarean section due to macrosomia. He was well at birth with a birth weight of $5.36 \mathrm{~kg}$ (99th centile for population). He was naturally conceived and there were no concerns during pregnancy. He was noted to have features of BWS (macroglossia (Fig. 1), hepatomegaly and single earlobe crease) post-delivery. Hypoglycaemia was noted at $2 \mathrm{~h}$ of life that persisted despite treatment with high dose intravenous glucose $(12 \mathrm{mg} / \mathrm{kg} / \mathrm{min})$.

\section{Investigation}

Hypoglycaemia screen performed on day 2 of life confirmed non-ketotic hyperinsulinemic hypoglycaemia with plasma insulin of $97 \mathrm{pmol} / \mathrm{L}$ at blood glucose of $1.6 \mathrm{mmol} / \mathrm{L}$ with low $3 \beta$-hydroxybutyrate $(<23 \mu \mathrm{mol} / \mathrm{L})$ and low non-esterified free fatty acids $(<176 \mu \mathrm{mol} / \mathrm{L})$.

Genetic analysis for mutations of $A B C C 8$ and KCNJ11 were negative. Genetic analysis for BWS by multiplex ligation-dependent probe amplification (MLPA)

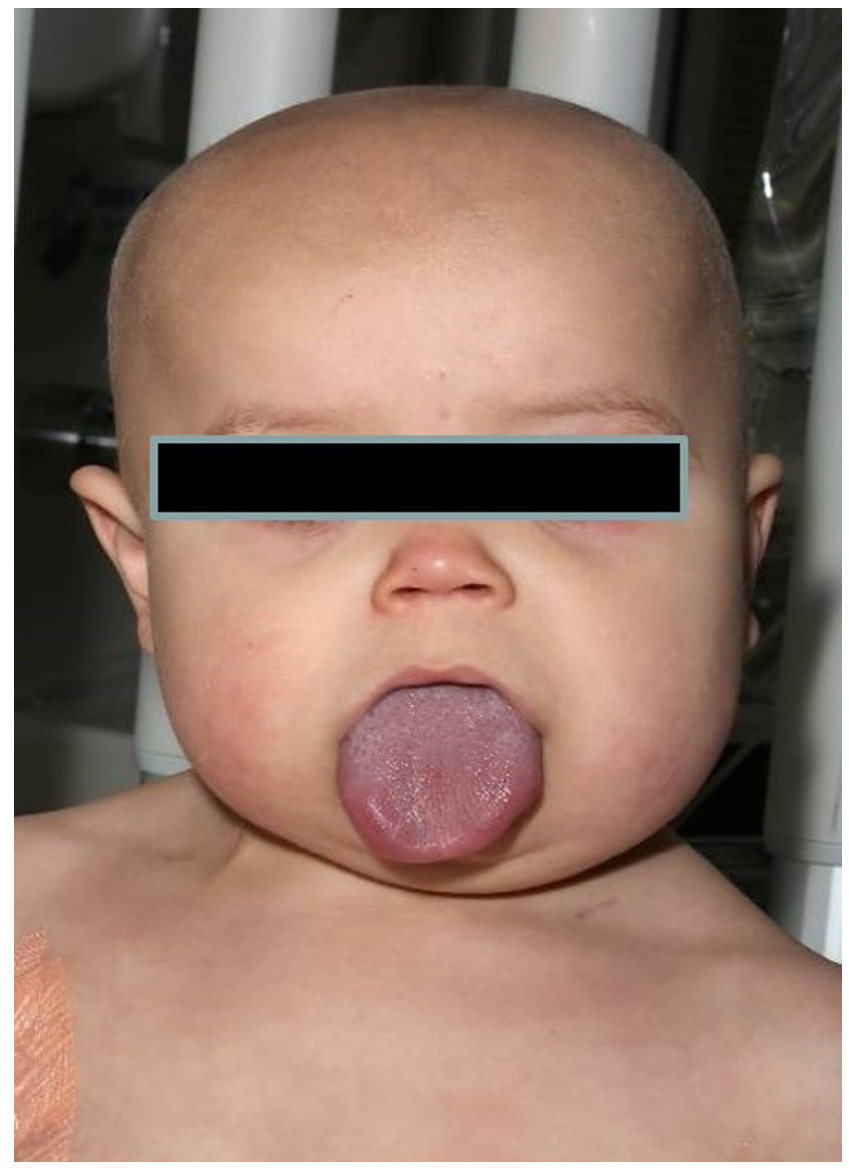

Figure 1

Macroglossia.

revealed hypomethylation at KCNQ1OT1:TSS-DMR and hypermethylation at the H19/IGF2:IG-DMR consistent with mosaic segmental paternal isodisomy of the $11 \mathrm{p} 15$ region (UPD(11)pat) without copy number changes.

Abdominal ultrasound was performed on day 4 of life to screen for Wilms' tumour and hepatoblastoma, as per international consensus guidelines (3). Ultrasound revealed a hypoechoic, focal ovoid mass measuring $29 \times 13 \times 12 \mathrm{~mm}$ in the left lobe of liver (segment 2 ). Also noted was bilateral renal enlargement with normal contour and echogenicity (right kidney $=6.0 \mathrm{~cm}$, left kidney $=6.7 \mathrm{~cm}$ (upper limit of normal at term $=5.7 \mathrm{~cm}$ )). MRI abdomen confirmed the hepatic mass but revealed a non-specific appearance.

Ultrasound-guided biopsy of the hepatic mass and subsequent histopathological examination revealed appearance to be consistent with hepatoblastoma showing exclusively epithelial component.

Histopathology of the surgically resected section showed a poorly defined mass merging with background liver structures. The tumour was composed of epithelial 
elements arranged in chord and trabeculae (Fig. 2). Cytology was not typical of a well-differentiated foetal type as there was a degree of nuclear enlargement and pleomorphism. A proportion of cells showed vacuolation and clear cytoplasm. No mesenchymal or small cell component was seen. In view of the patient's young age, setting of BWS and the absence of a history of chronic liver disease, the tumour was considered to be hepatoblastoma of epithelial, crowed foetal subtype, with pre-treatment tumour extension (PRETEXT - International Childhood Liver Tumours Strategy Group) stage 1. There was no evidence of post-chemotherapy change in the histology specimen.

Follow-up MRI imaging of the renal enlargement at 3 months of age revealed further enlargement of the kidneys with a mass in the left renal hilum measuring $33 \times 25 \times 36 \mathrm{~mm}$ with enhancing multi-cystic appearance to the periphery and solid central, suggestive of Wilms' tumour.

Histopathological examination, obtained through biopsy of the mass, showed a tri-phasic tumour composed of mesenchymal, blastemal and epithelial elements (Fig. 2). Mesenchymal elements resembled primitive mesenchyme with no heterologous elements observed. Blastemal was seen as nodules of primitive round blue cells and epithelial areas showed tubules and
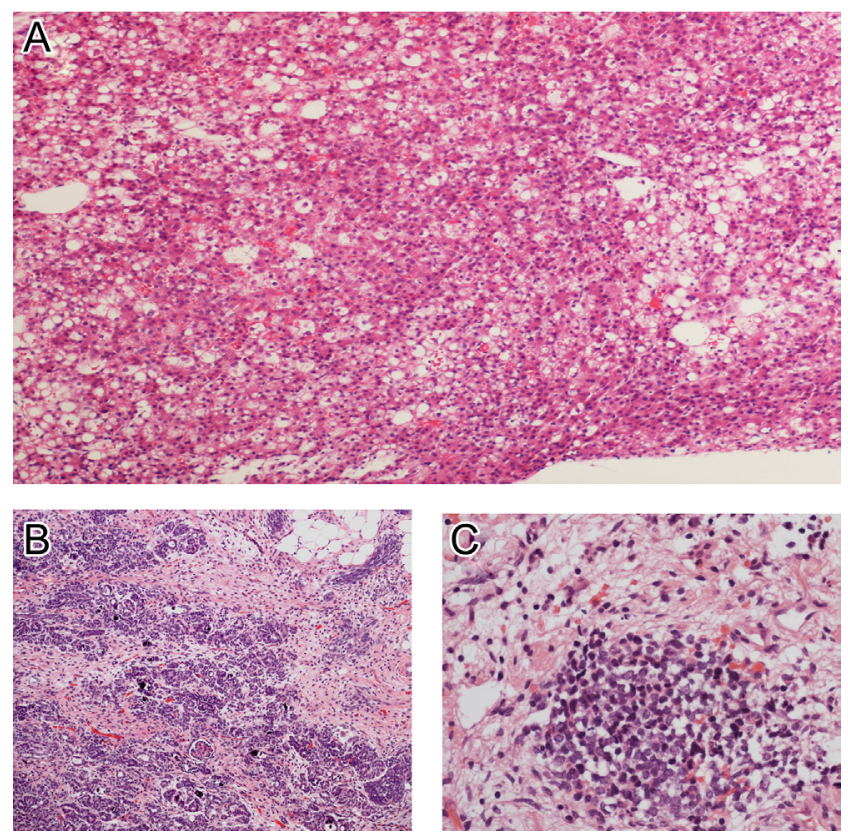

\section{Figure 2}

(A) Relatively well-differentiated hepatoid cells arranged in chords and tabaculae. There is clear vacuolated cytoplasm in a proportion of cells (glycogen). (B) Epithelial elements of Wilms' tumour seen as tubules and glomeruloid bodies. (C) Blastemal element of Wilms' tumour seen as nodule of undifferentiated round blue cells. glomeruloid structures (Fig. 2). No anaplasia was seen. On immunohistochemistry the tumour was positive for neural cell adhesion molecule (CD56) and Wilms' tumour protein (WT1). Overall features were typical of Wilms' tumour.

Histopathology of the resected specimen confirmed poor response to chemotherapy with only $20 \%$ of cells showing chemotherapy-induced change.

\section{Treatment}

Initially, the patient was stabilised on high concentration intravenous glucose infusion and intravenous glucagon infusion $(12 \mu \mathrm{g} / \mathrm{kg} / \mathrm{h})$. Diazoxide at $5 \mathrm{mg} / \mathrm{kg} /$ day in three divided doses was started and the dose was gradually increased. Hypoglycaemic episodes persisted despite increasing diazoxide to a maximum dose of $15 \mathrm{mg} / \mathrm{kg} / \mathrm{day}$. He was therefore commenced on subcutaneous octreotide at $5 \mu \mathrm{g} / \mathrm{kg} /$ day in three divided doses. The dose was gradually increased to $20 \mu \mathrm{g} / \mathrm{kg} /$ day, which permitted a reduction and eventual cessation of intravenous dextrose and glucagon. He was subsequently switched to once monthly $30 \mathrm{mg}$ deep subcutaneous Lanreotide injections at 1 year of age with good effect. He has good glycaemic control as established by an age-appropriate fast.

Treatment of the hepatoblastoma was commenced as per the Children's Cancer and Leukaemia Group (CCLG) hepatoblastoma treatment guidelines 2015 with four pre-operative cycles of cisplatin chemotherapy to reduce the size of tumour and facilitate surgical resection. He tolerated chemotherapy well but showed no response in terms of shrinkage of tumour size. He underwent resection of the liver lesion at 3 months of age.

Following the diagnosis of Wilms' tumour, the patient was commenced on 4 weeks of induction chemotherapy with vincristine and actinomycin D, as per CCLG Wilms' tumour guidelines 2016. Reassessment with MRI abdomen after 4 weeks revealed no response to chemotherapy and an increase in the extra renal growth. He subsequently underwent a nephrectomy at 5 months of age. Noting the poor initial response, post-operative chemotherapy was escalated to include cycles of cyclophosphamide with doxorubicin and carboplatin with etoposide. He underwent radiotherapy with seven fractions (1.5 Gy per fraction) at a total dose of 10.8 Gy.

\section{Outcome and follow-up}

An end-of-treatment MRI abdomen showed no evidence of tumour recurrence in the liver or kidney. 
Table 1 Trend in alpha-fetoprotein (AFP) in the first year of life.

\begin{tabular}{|c|c|c|}
\hline \multirow[b]{2}{*}{ Age (days) } & \multicolumn{2}{|c|}{$\operatorname{AFP}(I U / m L)$} \\
\hline & Values & Normal range* \\
\hline 4 & 128729 & $4396-91008$ \\
\hline 73 & 13207 & $5-867$ \\
\hline 118 & 1713 & $2.5-346$ \\
\hline 177 & 213 & $1-107$ \\
\hline 378 & 15 & $0.7-72$ \\
\hline
\end{tabular}

\section{Comment}

At diagnosis of hepatoblastoma

Pre-operative (hepatoblastoma resection)

Post-operative (hepatoblastoma resection)

Follow-up at 6 months

Follow-up at 1 year

*Serum AFP values adapted from Blohm et al. Klinische Padiatre 1991203246.

Serial measurements of alpha-fetoprotein (AFP) have progressively declined and are within the normal range for his age (Table 1). Blood glucose is currently stable on monthly $30 \mathrm{mg}$ Lanreotide injections. His growth is consistently following the 75th centile. He continues on oral feeds with input from the speech and language specialists.

\section{Discussion}

CHI is a common association with BWS (4). The cause of hyperinsulinism in BWS has not been fully elucidated. The genetic mutations responsible for the most common instances of CHI include ABCC 8 and KCNJ11 (located on chromosome $11 \mathrm{p} 15.1$, in close proximity to the BWS Locus). These two genes encode the two components of the pancreatic $\beta$-cell ATP-sensitive potassium channel. Mutations in these genes are inherited in autosomal dominant or recessive pattern. The recessively inherited defects are usually resistant to diazoxide therapy. Although mutations in ABCC8 and KCNJ11 have not been directly implicated as the cause of hyperinsulinism in BWS, the arrangement of genes in close proximity and the frequent combination of phenotypic features in mutant cases makes it likely that this close proximity to the imprinting centres associated with BWS affects their expression.

Diazoxide is the first-line medical management of CHI. It acts by blocking sulphonylurea receptors on pancreatic $\beta$-cells, which results in opening of the K-ATP channels and reduced insulin release. Diazoxide unresponsiveness is associated with the abolition of the function of pancreatic $\beta$-cell ATP-sensitive potassium channel. Patients with BWS show a varying degree of response to diazoxide (5). Somatostatin analogues (e.g. octreotide) are insulin inhibitors and the recognised second-line medical management of hyperinsulinemia in diazoxide unresponsive patients. The use of somatostatin analogues in persistent $\mathrm{CHI}$ has been shown to be effective and is well tolerated (6). Qualitative research has shown improved quality of life for parents of patients switched to long-acting somatostatin analogues due to lesser number of injections and reduced anxiety regarding missed or delayed injections (6).

Phenotypic features of lateralised overgrowth and tumour genesis in BWS spectrum have been well correlated with the underlying molecular defect in patients but the degree of severity of hyperinsulinism has not been well correlated. Interestingly, a previous report of patients requiring Lanreotide for diazoxide resistant severe hyperinsulinism in BWS also shared the same genotype of mosaic UPD(11)pat similar to our patient (7). Hence this genotype may be a pointer to diazoxide unresponsive persistent $\mathrm{CHI}$ which could respond well to somatostatin analogues.

The overall risk of tumour development in children with BWS is higher in those less than 4 years of age, with $95 \%$ of tumours occurring before 8 years of age (8). The tumour related presentation of most patients correlates with the BWS molecular subgroup, with segmental UPD(11)pat and Imprinting Centre 1 gain of methylation (IC1-GOM) having a higher tumour risk. Early hepatoblastoma in the late neonatal period has been reported in an infant with severe phenotype and UPD(11)pat, with a normal AFP recorded in the first week but rapidly rising in early infancy (9). In our case, hepatomegaly and raised AFP was detectable on day 5 of life, indicating congenital hepatoblastoma. The presence of severe hyperinsulinism contributed to early BWS surveillance and tumour detection. The histological appearance was not that of a typical hepatoblastoma, rather a crowded foetal type tumour. A further unusual feature was its lack of response to reductive chemotherapy as recommended in the CCLG guidelines.

The strong relation between Wilms' tumour and BWS may be because of the common factors of genomic imprinting and lesions at $11 \mathrm{p} 15$. The prevalence of Wilms' tumour varies significantly depending upon the molecular defect associated with the condition. A recent large meta- 
analysis has shown the mean age for development of Wilms' tumour in patients with BWS is 28 months (10). It is relatively uncommon for BWS patients to develop Wilms' tumour so early in infancy as in our case. Often Wilms' tumours respond well to standard chemotherapy but as was observed in relation to the hepatoblastoma, treatment response was poor with less than one quarter of cells showing chemotherapy response.

We report a rare case of BWS with diazoxide-resistant $\mathrm{CHI}$, hepatic neoplasm and Wilms' tumour, all presenting within the first 3 months of life. Early diagnosis and prompt screening enabled diagnosis of tumour at a relatively early stage. Earlier identified tumours may be more resistant to standard chemotherapy than tumours appearing in later childhood years. The early appearance and rapid progression of tumour in this case might necessitate a more frequent screening protocol with frequent abdominal ultrasound in UPD(11)pat genotype patients than the currently recommended 3 monthly schedule.

\section{Declaration of interest}

The authors declare that there is no conflict of interest that could be perceived as prejudicing the impartiality of the research reported.

\section{Funding}

This research did not receive any specific grant from any funding agency in the public, commercial or not-for-profit sector.

\section{Patient consent}

Consent to publication confirmed with parents by S S.

\section{Author contribution statement}

$\mathrm{S} U$ wrote the first draft, M D, R S and J H helped to revise the draft, J B prepared the final draft, S S revised the final draft and supervised the project.

\section{References}

1 Mussa A, Russo S, De Crescenzo A, Freschi A, Calzari L, Maitz S, Macchiaiolo M, Molinatto C, Baldassarre G, Mariani M, et al. (Epi)genotype-phenotype correlations in Beckwith-Wiedemann syndrome. European Journal of Human Genetics 201624 183-190. (https://doi.org/10.1038/ejhg.2015.88)

2 Maas SM, Vansenne F, Kadouch DJ, Ibrahim A, Bliek J, Hopman S, Mannens MM, Merks JH, Maher ER \& Hennekam RC. Phenotype, cancer risk, and surveillance in Beckwith-Wiedemann syndrome depending on molecular genetic subgroups. American Journal of Medical Genetics Part A 2016170 2248-2260. (https://doi. org/10.1002/ajmg.a.37801)

3 Brioude F, Kalish JM, Mussa A, Foster AC, Bliek J, Ferrero GB, Boonen SE, Cole T, Baker R, Bertoletti M, et al. Expert consensus document: Clinical and molecular diagnosis, screening and management of Beckwith-Wiedemann syndrome: an international consensus statement. Nature Reviews Endocrinology 201814 229-249. (https://doi.org/10.1038/nrendo.2017.166)

4 Spivey PS \& Bradshaw WT. Recognition and management of the infant with Beckwith-Wiedemann syndrome. Advances in Neonatal Care 20099 279-284; quiz 285. (https://doi.org/10.1097/ ANC.0b013e3181c2003f)

5 Menni F, De Lonlay P, Sevin C, Touati G, Peigné C, Barbier V, NihoulFékété C, Saudubray JM \& Robert JJ. Neurologic outcomes of 90 neonates and infants with persistent hyperinsulinemic hypoglycemia. Pediatrics 2001107 476-479. (https://doi.org/10.1542/peds.107.3.476)

6 Le Quan Sang KH, Arnoux JB, Mamoune A, Saint-Martin C, BellanneChantelot C, Valayannopoulos V, Brassier A, Kayirangwa H, Barbier V, Broissand C, et al. Successful treatment of congenital hyperinsulinism with long-acting release octreotide. European Journal of Endocrinology 2012166 333-339. (https://doi.org/10.1530/EJE-11-0874)

7 Al-Zubeidi H, Gottschalk ME \& Newfield RS. Successful use of long acting octreotide in two cases with Beckwith-Wiedemann syndrome and severe hypoglycaemia. International Journal of Pediatric Endocrinology 20142014 18. (https://doi.org/10.1186/1687-9856-2014-18)

8 Weksberg R, Shuman C \& Beckwith JB. Beckwith-Wiedemann syndrome. European Journal of Human Genetics 201018 8-14. (https:// doi.org/10.1038/ejhg.2009.106)

9 Mussa A, Ferrero GB, Ceoloni B, Basso E, Chiesa N, De Crescenzo A, Pepe E, Silengo M \& De Sanctis L. Neonatal hepatoblastoma in a newborn with severe phenotype of Beckwith-Wiedemann syndrome. European Journal of Pediatrics 2011170 1407-1411. (https://doi. org/10.1007/s00431-011-1455-0)

10 Maas SM, Vansenne F, Kadouch DJM, Ibrahim A, Bliek J, Hopman S, Mannens MM, Merks JH, Maher ER \& Hennekam RC. Phenotype, cancer risk, and surveillance in Beckwith-Wiedemann syndrome depending on molecular genetic subgroups. American Journal of Medical Genetics Part A 2016170 2248-2260. (https://doi. org/10.1002/ajmg.a.37801)

Received in final form 30 January 2019 Accepted 11 February 2019 